QUARTERLY OF APPLIED MATHEMATICS

VOLUME LXIX, NUMBER 2

JUNE 2011, PAGES 317-330

S 0033-569X(2011)01219-3

Article electronically published on March 9, 2011

\title{
A MULTI-DIMENSIONAL BLOW-UP PROBLEM DUE TO A CONCENTRATED NONLINEAR SOURCE IN $\mathbb{R}^{N}$
}

\author{
BY \\ C. Y. CHAN (Department of Mathematics, University of Louisiana at Lafayette, Lafayette, \\ Louisiana 70504-1010) \\ AND \\ P. TRAGOONSIRISAK (Department of Mathematics and Computer Science, Fort Valley State \\ University, Fort Valley, Georgia 31030)
}

Abstract. Let $B$ be an $N$-dimensional ball $\left\{x \in \mathbb{R}^{N}:|x|<R\right\}$ centered at the origin with a radius $R$, and $\partial B$ be its boundary. Also, let $\nu(x)$ denote the unit inward normal at $x \in \partial B$, and let $\chi_{B}(x)$ be the characteristic function, which is 1 for $x \in B$, and 0 for $x \in \mathbb{R}^{N} \backslash B$. This article studies the following multi-dimensional semilinear parabolic problem with a concentrated nonlinear source on $\partial B$ :

$$
\begin{gathered}
u_{t}-\triangle u=\alpha \frac{\partial \chi_{B}(x)}{\partial \nu} f(u) \text { in } \mathbb{R}^{N} \times(0, T], \\
u(x, 0)=\psi(x) \text { for } x \in \mathbb{R}^{N}, u(x, t) \rightarrow 0 \text { as }|x| \rightarrow \infty \text { for } 0<t \leq T,
\end{gathered}
$$

where $\alpha$ and $T$ are positive numbers, $f$ and $\psi$ are given functions such that $f(0) \geq 0$, $f(u)$ and $f^{\prime}(u)$ are positive for $u>0, f^{\prime \prime}(u) \geq 0$ for $u>0$, and $\psi$ is nontrivial on $\partial B$, nonnegative, and continuous such that $\psi \rightarrow 0$ as $|x| \rightarrow \infty, \int_{\mathbb{R}^{N}} \psi(x) d x<\infty$, and $\triangle \psi+\alpha\left(\partial \chi_{B}(x) / \partial \nu\right) f(\psi(x)) \geq 0$ in $\mathbb{R}^{N}$. It is shown that the problem has a unique nonnegative continuous solution before blowup occurs. We assume that $\psi(x)=M(0)>$ $\psi(y)$ for $x \in \partial B$ and $y \notin \partial B$, where $M(t)=\sup _{x \in \mathbb{R}^{N}} u(x, t)$. It is proved that if $u$ blows up in a finite time, then it blows up everywhere on $\partial B$. If, in addition, $\psi$ is radially symmetric about the origin, then we show that if $u$ blows up, then it blows up on $\partial B$ only. Furthermore, if $f(u) \geq \kappa u^{p}$, where $\kappa$ and $p$ are positive constants such that $p>1$, then it is proved that for any $\alpha, u$ always blows up in a finite time for $N \leq 2$; for $N \geq 3$, it is shown that there exists a unique number $\alpha^{*}$ such that $u$ exists globally for $\alpha \leq \alpha^{*}$ and blows up in a finite time for $\alpha>\alpha^{*}$. A formula for computing $\alpha^{*}$ is given.

1. Introduction. Let $H=\partial / \partial t-\triangle, T$ be a positive real number, $x=\left(x_{1}, x_{2}, \ldots\right.$, $\left.x_{N}\right)$ be a point in the $N$-dimensional Euclidean space $\mathbb{R}^{N}, \Omega=\mathbb{R}^{N} \times(0, T], B$ be an

Received October 12, 2009.

2000 Mathematics Subject Classification. Primary 35K60, 35K57, 35 B35.

Key words and phrases. Concentrated nonlinear source, Existence, Uniqueness, Blowup.

E-mail address: chan@louisiana.edu

E-mail address: tragoonsirisakp@fvsu.edu 
$N$-dimensional ball $\left\{x \in \mathbb{R}^{N}:|x-\bar{b}|<R\right\}$ centered at a given point $\bar{b}$ with a radius $R$, $\partial B$ be the boundary of $B, \nu(x)$ denote the unit inward normal at $x \in \partial B$, and

$$
\chi_{B}(x)=\left\{\begin{array}{l}
1 \text { for } x \in B \\
0 \text { for } x \in \mathbb{R}^{N} \backslash B
\end{array}\right.
$$

be the characteristic function. Without loss of generality, let $\bar{b}$ be the origin. We would like to study the following multi-dimensional semilinear parabolic problem with a source on the surface of the ball:

$$
\left.\begin{array}{l}
H u=\alpha \frac{\partial \chi_{B}(x)}{\partial \nu} f(u) \text { in } \Omega, \\
x \in \mathbb{R}^{N}, u(x, t) \rightarrow 0 \text { as }|x| \rightarrow \infty \text { for } 0<t \leq T
\end{array}\right\}
$$

where $\alpha$ is a positive constant, $f$ and $\psi$ are given functions such that $f(0) \geq 0, f(u)$ and $f^{\prime}(u)$ are positive for $u>0, f^{\prime \prime}(u) \geq 0$ for $u>0$, and $\psi$ is nontrivial on $\partial B$, nonnegative, and continuous such that $\psi \rightarrow 0$ as $|x| \rightarrow \infty, \int_{\mathbb{R}^{N}} \psi(x) d x<\infty$, and $\triangle \psi+\alpha\left(\partial \chi_{B}(x) / \partial \nu\right) f(\psi(x)) \geq 0$ in $\mathbb{R}^{N}$. We note that such a problem in a bounded domain, instead of $\mathbb{R}^{N}$, was studied by Chan and Tian ([2], [3]).

A solution $u$ is said to blow up at the point $\left(x, t_{b}\right)$ if there exists a sequence $\left\{\left(x_{n}, t_{n}\right)\right\}$ such that $u\left(x_{n}, t_{n}\right) \rightarrow \infty$ as $\left(x_{n}, t_{n}\right) \rightarrow\left(x, t_{b}\right)$.

In Section 2, we show that the nonlinear integral equation corresponding to the problem (1.1) has a unique nonnegative continuous solution $u$, which is a nondecreasing function of $t$. We then prove that $u$ is the unique solution of the problem (1.1). Let $M(t)$ denote $\sup _{x \in \mathbb{R}^{N}} u(x, t)$. We assume that

$$
\psi(x)=M(0)>\psi(y) \text { for } x \in \partial B \text { and } y \notin \partial B .
$$

If $t_{b}$ is finite, we show that $u$ blows up everywhere on $\partial B$. If, in addition, $\psi$ is radially symmetric about the origin, then we prove that if $u$ blows up, then it blows up everywhere on $\partial B$ only. Let $\kappa$ and $p$ be positive constants such that $p>1$. If $f(u) \geq \kappa u^{p}$, then we prove, in Section 3, that for any $\alpha, u$ always blows up in a finite time if $N \leq 2$. This behavior is completely different from that for $N \geq 3$. In Section 4 , we show that for $N \geq 3$, there exists a unique number $\alpha^{*}$ such that $u$ exists globally for $\alpha \leq \alpha^{*}$ and blows up in a finite time for $\alpha>\alpha^{*}$. We also derive a formula for computing $\alpha^{*}$. We note that whether a solution of the heat equation without a concentrated source in an unbounded domain blows up in a finite time was studied by Fujita [7, and Pinsky [8].

2. Existence, uniqueness, and blowup. To derive the integral equation from the problem (1.1), we use the adjoint operator $(-\partial / \partial t-\triangle)$ of $H$. Using Green's second identity, we obtain

$$
u(x, t)=\int_{\mathbb{R}^{N}} g(x, t ; \xi, 0) \psi(\xi) d \xi+\alpha \int_{0}^{t} \int_{\mathbb{R}^{N}} g(x, t ; \xi, \tau) \frac{\partial \chi_{B}(\xi)}{\partial \nu} f(u(\xi, \tau)) d \xi d \tau,
$$

where

$$
g(x, t ; \xi, \tau)=\left\{\begin{array}{l}
\frac{1}{[4 \pi(t-\tau)]^{N / 2}} \exp \left(-\frac{|x-\xi|^{2}}{4(t-\tau)}\right), t>\tau \\
0, t<\tau
\end{array}\right.
$$


is Green's function (cf. Stakgold [10, p. 198]) corresponding to the problem (1.1). Using integration by parts and the Divergence Theorem, we have

$$
u(x, t)=\int_{\mathbb{R}^{N}} g(x, t ; \xi, 0) \psi(\xi) d \xi+\alpha \int_{0}^{t} \int_{\partial B} g(x, t ; \xi, \tau) f(u(\xi, \tau)) d S_{\xi} d \tau
$$

(cf. Chan and Tragoonsirisak [4]). We note that $\int_{\mathbb{R}^{N}} \psi(x) d x<\infty$ is used to show that the first term on the right-hand side is continuous, and Lemma 2.1 of Chan and Tragoonsirisak [4] is used to show that the second term is continuous for $t>0$.

To establish the next two results, we modify the techniques in proving Theorems 3 and 4 of Chan and Tian 2 2 for a blow-up problem in a bounded domain, and Theorems 2.1 and 2.2 of Chan and Tragoonsirisak [4] for a quenching problem in $\mathbb{R}^{N}$.

Theorem 2.1. There exists some $t_{b}$ such that for $0 \leq t<t_{b}$, the integral equation (2.2) has a unique continuous nonnegative solution $u$, and $u$ is a nondecreasing function of $t$. If $t_{b}$ is finite, then $u$ is unbounded in $\left[0, t_{b}\right)$.

Our next result shows that the solution of the integral equation (2.2) is the solution of the problem (1.1).

Theorem 2.2. The problem (1.1) has a unique solution $u$ for $0 \leq t<t_{b}$.

Henceforth, we assume that (1.2) holds. Our next result shows that at $t_{b}, u$ blows up everywhere on the surface of the ball.

THEOREM 2.3. If $t_{b}$ is finite, then at $t_{b}, u$ blows up everywhere on $\partial B$.

Proof. By Theorems 2.1 and 2.2, there exists some $t_{b}$ such that for $0 \leq t<t_{b}$, the problem (1.1) has a unique nonnegative continuous solution $u$, which is a nondecreasing function of $t$. Since $u(x, t)$ on $\partial B \times\left(0, t_{b}\right)$ is known, let us denote it by $\tilde{g}(x, t)$, and rewrite the problem (1.1) as two initial-boundary value problems:

$$
\begin{gathered}
\left.\begin{array}{c}
H u=0 \text { in } B \times\left(0, t_{b}\right), \\
u(x, 0)=\psi(x) \text { on } \bar{B}, u(x, t)=\tilde{g}(x, t) \text { on } \partial B \times\left(0, t_{b}\right) ;
\end{array}\right\} \\
H u=0 \text { in }\left(\mathbb{R}^{N} \backslash \bar{B}\right) \times\left(0, t_{b}\right), \\
\left.\begin{array}{c}
u(x, 0)=\psi(x) \text { on } \mathbb{R}^{N} \backslash B, u(x, t)=\tilde{g}(x, t) \text { on } \partial B \times\left(0, t_{b}\right), \\
u(x, t) \rightarrow 0 \text { as }|x| \rightarrow \infty \text { for } 0<t<t_{b} .
\end{array}\right\}
\end{gathered}
$$

Let us consider the problem (2.3). From the strong maximum principle (cf. Friedman [6. p. 34]), $u$ attains its maximum somewhere on $\partial B$ for $t>0$. For the problem (2.4), $u(x, t) \rightarrow 0$ as $|x| \rightarrow \infty$. Since $u$ is a nondecreasing function of $t$, it follows from the Phragmén-Lindelöf Principle (cf. Protter and Weinberger [9, pp. 183-185]) that $u$ attains its maximum somewhere on $\partial B$ for $t>0$. Thus for each given $\rho \in\left(0, t_{b}\right), u$ attains its maximum for $0 \leq t \leq \rho$ somewhere on $\partial B \times\{\rho\}$.

Suppose that there exists a smallest positive value of $t$, say $t_{1}$, and some $\bar{y} \notin \partial B$ such that $u\left(\bar{y}, t_{1}\right)=\min _{x \in \partial B} u\left(x, t_{1}\right)$. We claim that for $x \in \partial B, u\left(x, t_{1}\right)=u\left(\bar{y}, t_{1}\right)$. If this is not true, then there exists some $\bar{x} \in \partial B$ such that $u\left(\bar{x}, t_{1}\right)>\min _{x \in \partial B} u\left(x, t_{1}\right)$. Since $u$ is continuous, there exists some point $\left(\tilde{y}, t_{1}\right)$ in a neighborhood of $\left(\bar{x}, t_{1}\right)$ such that $\tilde{y} \notin \partial B$ and $u\left(\tilde{y}, t_{1}\right)>\min _{x \in \partial B} u\left(x, t_{1}\right)$. This contradicts the definition of $t_{1}$. Thus, $u$ attains 
its maximum at $\left(\bar{y}, t_{1}\right)$ for $0 \leq t \leq t_{1}$. If $\bar{y} \in B$, then it follows from the strong maximum principle and the continuity of $u$ that $u \equiv u\left(\bar{y}, t_{1}\right)$ on $\bar{B} \times\left[0, t_{1}\right]$. This contradicts (1.2). If $\bar{y} \in\left(\mathbb{R}^{N} \backslash \bar{B}\right)$, then let $\tilde{B}$ be an $N$-dimensional ball $\left\{x \in \mathbb{R}^{N}:|x|<\tilde{R}\right\}$ such that $\bar{y} \in \tilde{B}$. By the strong maximum principle and the continuity of $u, u \equiv u\left(\bar{y}, t_{1}\right)$ on $(\tilde{B} \backslash B) \times\left[0, t_{1}\right]$. Again, this contradicts (1.2). Thus for any $t>0$,

$$
u(x, t)>u(y, t) \text { for any } x \in \partial B \text { and any } y \notin \partial B .
$$

We claim that for each $t>0, u$ attains the same value for $x \in \partial B$. If this is not true, then for some $t>0$, there exists some $\tilde{x} \in \partial B$ such that $u(\tilde{x}, t)>\min _{x \in \partial B} u(x, t)$. By continuity, there exists some point $(\hat{y}, t)$ in a neighborhood of $(\tilde{x}, t)$ such that $\hat{y} \notin \partial B$ and $u(\hat{y}, t)>\min _{x \in \partial B} u(x, t)$. This contradicts (2.5). Hence for any $t>0$,

$$
u(x, t)=M(t) \text { for } x \in \partial B, M(t)>u(y, t) \text { for any } y \notin \partial B .
$$

This implies that for each $t>0, u$ attains its absolute maximum on $\partial B$. Thus, if $u$ blows up, then it blows up there. Since $t_{b}$ is finite, it follows from Theorem 2.1 that $u$ blows up everywhere on $\partial B$.

Our next result shows that for the symmetric case, $u$ blows up on $\partial B$ only.

THEOREM 2.4. Under the additional assumption that $\psi$ is radially symmetric about the origin, if $t_{b}$ is finite, then at $t_{b}, u$ blows up on $\partial B$ only.

Proof. Let us construct a sequence $\left\{u_{n}\right\}$ in $\Omega$ by $u_{0}(x, t)=\psi(x)$, and for $n=$ $0,1,2, \ldots$,

$$
\begin{gathered}
H u_{n+1}=\alpha \frac{\partial \chi_{B}(x)}{\partial \nu} f\left(u_{n}\right) \text { in } \Omega, \\
u_{n+1}(x, 0)=\psi(x) \text { for } x \in \mathbb{R}^{N}, u_{n+1}(x, t) \rightarrow 0 \text { as }|x| \rightarrow \infty \text { for } 0<t \leq T .
\end{gathered}
$$

From (2.2),

$$
u_{n+1}(x, t)=\int_{\mathbb{R}^{N}} g(x, t ; \xi, 0) \psi(\xi) d \xi+\alpha \int_{0}^{t} \int_{\partial B} g(x, t ; \xi, \tau) f\left(u_{n}(\xi, \tau)\right) d S_{\xi} d \tau .
$$

We note that $\triangle \psi+\alpha\left(\partial \chi_{B}(x) / \partial \nu\right) f(\psi(x)) \geq 0$ in $\mathbb{R}^{N}$. Thus,

$$
\begin{gathered}
H\left(u_{1}-u_{0}\right) \geq \alpha \frac{\partial \chi_{B}(x)}{\partial \nu}\left(f\left(u_{0}(x, t)\right)-f(\psi(x))\right)=0 \text { in } \Omega, \\
\left(u_{1}-u_{0}\right)(x, 0)=0 \text { for } x \in \mathbb{R}^{N},\left(u_{1}-u_{0}\right)(x, t) \rightarrow 0 \text { as }|x| \rightarrow \infty \text { for } 0<t \leq T .
\end{gathered}
$$

Since $g(x, t ; \xi, \tau)>0$ in $\left\{(x, t ; \xi, \tau): x\right.$ and $\xi$ are in $\left.\mathbb{R}^{N}, T \geq t>\tau \geq 0\right\}$, it follows from (2.2) that $u_{1}(x, t) \geq u_{0}(x, t)$ in $\Omega$. Let us assume that for some positive integer $j$, $\psi \leq u_{1} \leq u_{2} \leq u_{3} \leq \cdots \leq u_{j-1} \leq u_{j}$ in $\Omega$. We have

$$
\begin{gathered}
H\left(u_{j+1}-u_{j}\right)=\alpha \frac{\partial \chi_{B}(x)}{\partial \nu}\left(f\left(u_{j}\right)-f\left(u_{j-1}\right)\right) \text { in } \Omega, \\
\left(u_{j+1}-u_{j}\right)(x, 0)=0 \text { for } x \in \mathbb{R}^{N},\left(u_{j+1}-u_{j}\right)(x, t) \rightarrow 0 \text { as }|x| \rightarrow \infty \text { for } 0<t \leq T .
\end{gathered}
$$

Since $f$ is an increasing function and $u_{j} \geq u_{j-1}$, we have $f\left(u_{j}\right)-f\left(u_{j-1}\right) \geq 0$. It follows from (2.2) that $u_{j+1} \geq u_{j}$. By the principle of mathematical induction,

$$
\psi \leq u_{1} \leq u_{2} \leq \cdots \leq u_{n-1} \leq u_{n} \text { in } \Omega .
$$


Since $u_{n}$ is an increasing sequence as $n$ increases, it follows from the Monotone Convergence Theorem that we have (2.2) with $\lim _{n \rightarrow \infty} u_{n}(x, t)=u(x, t)$.

Since $\psi(x)$ is radially symmetric about the origin, namely $\psi(x)=\psi(|x|)$, it follows from (2.1) and the construction (2.7) that

$$
\begin{aligned}
u_{1}(x, t)= & \frac{1}{(4 \pi t)^{N / 2}} \int_{\mathbb{R}^{N}} \exp \left(-\frac{|x-\xi|^{2}}{4 t}\right) \psi(|\xi|) d \xi \\
& +\alpha \int_{0}^{t} \frac{1}{[4 \pi(t-\tau)]^{N / 2}} \int_{\partial B} \exp \left(-\frac{|x-\xi|^{2}}{4(t-\tau)}\right) f(\psi(|\xi|)) d S_{\xi} d \tau \\
= & \frac{1}{(4 \pi t)^{N / 2}} \lim _{r \rightarrow \infty} \int_{B(0, r)} \exp \left(-\frac{|x-\xi|^{2}}{4 t}\right) \psi(|\xi|) d \xi \\
& +\alpha \int_{0}^{t} \frac{1}{[4 \pi(t-\tau)]^{N / 2}} \int_{\partial B} \exp \left(-\frac{|x-\xi|^{2}}{4(t-\tau)}\right) f(\psi(|\xi|)) d S_{\xi} d \tau
\end{aligned}
$$

where $B(0, r)$ is the $N$-dimensional ball centered at the origin with a radius $r$. Thus, $u_{1}(x, t)$ is radially symmetric about the origin. We assume that for some positive integer $j, u_{j}(x, t)$ is radially symmetric about the origin, namely $u_{j}(x, t)=u_{j}(|x|, t)$. Then,

$$
\begin{aligned}
u_{j+1}(x, t)= & \frac{1}{(4 \pi t)^{N / 2}} \lim _{r \rightarrow \infty} \int_{B(0, r)} \exp \left(-\frac{|x-\xi|^{2}}{4 t}\right) \psi(|\xi|) d \xi \\
& +\alpha \int_{0}^{t} \frac{1}{[4 \pi(t-\tau)]^{N / 2}} \int_{\partial B} \exp \left(-\frac{|x-\xi|^{2}}{4(t-\tau)}\right) f\left(u_{j}(|\xi|, \tau)\right) d S_{\xi} d \tau
\end{aligned}
$$

is also radially symmetric about the origin. By the principle of mathematical induction, $u_{n}(x, t)$ is radially symmetric about the origin for $n=0,1,2, \ldots$. Hence, $u(x, t)=$ $\lim _{n \rightarrow \infty} u_{n}(x, t)$ is radially symmetric about the origin.

From the problem (2.4), we have

$$
\begin{gathered}
u_{t}-\left(u_{r r}+\frac{N-1}{r} u_{r}\right)=0 \text { in }(R, \infty) \times\left(0, t_{b}\right), \\
u(r, 0)=\psi(r) \text { on }[R, \infty), \\
u(R, t)=M(t), u(r, t) \rightarrow 0 \text { as } r \rightarrow \infty \text { for } 0<t<t_{b} .
\end{gathered}
$$

From Theorem 2.1, $u_{t}(x, t) \geq 0$ in $\left(\mathbb{R}^{N} \backslash \bar{B}\right) \times\left(0, t_{b}\right)$. Thus,

$$
u_{r r}+\frac{N-1}{r} u_{r}=u_{t} \geq 0 .
$$

We note from (2.6) and the parabolic version of Hopf's lemma (cf. Friedman [6, p. 49]) that $u_{r}(R, t)<0$ for $0<t<t_{b}$. Hence for $0<t<t_{b}, \lim _{r \rightarrow R^{+}} u_{r r}(r, t) \geq 0$ for $N \geq 1$. Therefore, if $u$ blows up, then it blows up on $\partial B$ only.

For the problem (2.3), it follows from Theorem 2.1 that $u_{t}(x, t) \geq 0$ in $B \times\left(0, t_{b}\right)$. By Corollary 2 of Friedman [6. p. 74], $u$ is infinitely differentiable. Hence, $H u_{t}=0$ in $B \times\left(0, t_{b}\right)$. If $u_{t}=0$ somewhere in $B \times\left(0, t_{b}\right)$, say at $t=t_{2}$, then it follows from the problem (2.3) and the strong maximum principle that $u_{t} \equiv 0$ in $B \times\left(0, t_{2}\right]$, and hence $u(x, t)=\psi(x)$ for $(x, t) \in B \times\left(0, t_{2}\right]$. By continuity, we have for $(x, t) \in \partial B \times\left[0, t_{2}\right]$, $u(x, t)=\psi(x)=M(0)$, which is bounded. Since the solution $u$ is continuous on 
$\partial B \times\left[0, t_{b}\right)$, there exists some $t_{3}\left(\geq t_{2}\right)$ such that $u_{t}>0$ in $B \times\left[t_{3}, t_{b}\right)$. Because $u$ is radially symmetric, we have

$$
\begin{gathered}
u_{t}-\left(u_{r r}+\frac{N-1}{r} u_{r}\right)=0 \text { in }(0, R) \times\left(0, t_{b}\right), \\
u(r, 0)=\psi(r) \text { on }[0, R], \\
u_{r}(0, t)=0, u(R, t)=M(t) \text { for } 0<t<t_{b} .
\end{gathered}
$$

Thus,

$$
u_{r r}+\frac{N-1}{r} u_{r}=u_{t}>0
$$

in $B \times\left[t_{3}, t_{b}\right)$. Since $\lim _{r \rightarrow 0} u_{r r}+(N-1) \lim _{r \rightarrow 0}\left(u_{r} / r\right)=N u_{r r}(0, t)$, we have $u_{r r}(0, t)>$ 0 , implying that $u$ is concave up near the origin $r=0$. Because $u_{r}(0, t)=0$ for $t_{3} \leq t<t_{b}$, we have $u_{r}>0$ near the origin for $t_{3} \leq t<t_{b}$. We would like to show that $u(0, t)$ is bounded as $t$ tends to $t_{b}$. Let us assume, on the contrary, that $u(0, t)$ tends to infinity as $t$ tends to $t_{b}$. If $u_{t}(0, t)$ is bounded, say by a constant $k_{1}$, then

$$
u(0, t) \leq u(0,0)+k_{1} t \text { for } 0<t<t_{b} .
$$

Because $u(0,0)$ is bounded, we have a contradiction. Thus, $u_{t}(0, t)$ tends to infinity as $t$ tends to $t_{b}$. Since $u_{t}(0, t)=N u_{r r}(0, t)$, we have $u_{r r}(0, t)$ tending to infinity as $t$ tends to $t_{b}$. Thus for $t_{3} \leq t<t_{b}$, there are points in a neighborhood of the origin $r=0$ with values larger than $u(0, t)$, and hence, $u$ should blow up before $t_{b}$. This contradicts the definition of $t_{b}$. Hence, $u(0, t)$ is bounded as $t$ tends to $t_{b}$. Next, we would like to show that the graph of $u$ is concave up near $\partial B$. Since $u(r, t)$ tends to infinity as $r$ tends to $R$ and $t$ tends to $t_{b}$, and $u$ is a strictly increasing function of $t \in\left[t_{3}, t_{b}\right)$, we have for any given number $M_{1}$ sufficiently large, that there exists $\tilde{r}$ sufficiently close to $R$ and some $\tilde{t}$ such that $u(r, t)>M_{1}$ for $r \in[\tilde{r}, R]$ and $t \in\left[\tilde{t}, t_{b}\right)$. We claim that for any given large number $M_{2}$, we can choose $\tilde{r}$ and $\tilde{t}$ such that $u_{t}(r, t)>M_{2}$ for $r \in[\tilde{r}, R]$ and $t \in\left[\tilde{t}, t_{b}\right)$. To prove this, let us assume that $u_{t}(r, t)$ is bounded, say by a constant $M_{2}$. Then, $u(r, t) \leq u(r, 0)+M_{2} t$. We note that for $M_{1}>u(R, 0)+M_{2} t_{b}$, we have $\tilde{r}$ sufficiently close to $R$ and some $\tilde{t}$ such that $u(r, t)>M_{1}$ for $r \in[\tilde{r}, R]$ and $t \in\left[\tilde{t}, t_{b}\right)$. Thus,

$$
u(r, t) \leq u(r, 0)+M_{2} t \leq u(R, 0)+M_{2} t_{b}<M_{1}
$$

for $r \in[\tilde{r}, R]$ and $t \in\left[\tilde{t}, t_{b}\right)$. We have a contradiction. Hence, $u_{t}(r, t)$ can be made as large as we please. By choosing $r$ and $t$ sufficiently close to $R$ and $t_{b}$ respectively, if $u_{r r}(r, t) \leq 0$, then it follows from (2.8) that $u_{r}(r, t)$ can be made as large as we please. This gives a contradiction to $u_{r r}(r, t) \leq 0$ since $u(r, t)$ can be made as large as we wish. Thus, $u$ is concave up near $\partial B$. Because $t_{b}$ is finite, it follows from Theorem 2.3 that $u$ blows up on $\partial B$ only.

3. $\boldsymbol{N} \leq \mathbf{2}$. In the sequel, we assume that $f(u) \geq \kappa u^{p}$, where $\kappa$ and $p$ are positive constants such that $p>1$. Let

$$
I(x, t)=\int_{\partial B} g(x, t ; \xi, 0) d S_{\xi} .
$$


Lemma 3.1 of Chan and Tragoonsirisak 4] states that for $t \geq 1$ and any $x \in \bar{B}$,

$$
(4 \pi)^{-N / 2} e^{-R^{2}} \omega_{N} R^{N-1} t^{-N / 2} \leq I(x, t) \leq(4 \pi)^{-N / 2} \omega_{N} R^{N-1} t^{-N / 2},
$$

where $\omega_{N}$ denotes the surface area of an $N$-dimensional unit sphere.

Theorem 3.1. If $N \leq 2$, then for any $\alpha$ and any $\psi(x)$, the solution $u$ of the problem (1.1) always blows up in a finite time.

Proof. Let

$$
h(x)=\frac{e^{-|x|^{2}}}{\pi^{N / 2}} .
$$

We note that $h(x)>0, h(x) \rightarrow 0$ as $|x| \rightarrow \infty$,

$$
\begin{gathered}
\int_{\mathbb{R}^{N}} h(x) d x=\int_{-\infty}^{\infty} \frac{e^{-x_{1}^{2}}}{\sqrt{\pi}} d x_{1} \cdots \int_{-\infty}^{\infty} \frac{e^{-x_{N}^{2}}}{\sqrt{\pi}} d x_{N}=1, \\
\int_{\partial B} h(x) d S_{x}=\frac{e^{-R^{2}}}{\pi^{N / 2}} \int_{\partial B} d S_{x}=\frac{e^{-R^{2}} \omega_{N} R^{N-1}}{\pi^{N / 2}}, \\
\int_{\bar{B}} h(x) d x<\int_{\mathbb{R}^{N}} h(x) d x=1 \\
\triangle h=\frac{4 e^{-|x|^{2}}|x|^{2}}{\pi^{N / 2}}-2 N h(x) \geq-2 N h(x) .
\end{gathered}
$$

Let

$$
F(t)=\int_{\mathbb{R}^{N}} u(x, t) h(x) d x
$$

Since $u$ is the solution of the problem (1.1), $F(t)$ may be regarded as a distribution. Thus,

$$
\begin{aligned}
F^{\prime}(t) & =\int_{\mathbb{R}^{N}} u_{t}(x, t) h(x) d x \\
& =\int_{\mathbb{R}^{N}}\left(\triangle u(x, t)+\alpha \frac{\partial \chi_{B}(x)}{\partial \nu} f(u(x, t))\right) h(x) d x \\
& \geq \int_{\mathbb{R}^{N}} \triangle u(x, t) h(x) d x+\alpha \kappa \int_{\mathbb{R}^{N}} \frac{\partial \chi_{B}(x)}{\partial \nu} u^{p}(x, t) h(x) d x \\
& =\int_{\mathbb{R}^{N}} \Delta u(x, t) h(x) d x+\alpha \kappa \int_{\partial B} u^{p}(x, t) h(x) d S_{x} .
\end{aligned}
$$


Using Green's second identity and (3.2), we have

$$
\begin{aligned}
\int_{\mathbb{R}^{N}} & \triangle u(x, t) h(x) d x \\
& =\lim _{\tilde{R} \rightarrow \infty} \int_{|x|<\tilde{R}} \triangle u(x, t) h(x) d x \\
& =\lim _{\tilde{R} \rightarrow \infty} \int_{|x|<\tilde{R}} u(x, t) \triangle h(x) d x \\
& =\int_{\mathbb{R}^{N}} u(x, t) \triangle h(x) d x \\
& \geq-2 N \int_{\mathbb{R}^{N}} u(x, t) h(x) d x \\
& =-2 N F(t) .
\end{aligned}
$$

From (2.6),

$$
F(t) \leq M(t) \int_{\mathbb{R}^{N}} h(x) d x=M(t)
$$

Thus,

$$
\begin{aligned}
\int_{\partial B} u^{p}(x, t) h(x) d S_{x} & =M^{p}(t) \int_{\partial B} h(x) d S_{x} \\
& \geq F^{p}(t) \int_{\partial B} h(x) d S_{x} \\
& =\frac{e^{-R^{2}} \omega_{N} R^{N-1} F^{p}(t)}{\pi^{N / 2}} .
\end{aligned}
$$

Hence,

$$
F^{\prime}(t)+2 N F(t) \geq \frac{\alpha \kappa e^{-R^{2}} \omega_{N} R^{N-1}}{\pi^{N / 2}} F^{p}(t) .
$$

Solving this Bernoulli inequality, we obtain

$$
F^{1-p}(t) \leq \frac{\alpha \kappa e^{-R^{2}} \omega_{N} R^{N-1}}{2 N \pi^{N / 2}}+C e^{2 N(p-1) t},
$$

where $C$ is to be determined. We can choose for $\tilde{t} \geq 0$,

$$
C=\left(F^{1-p}(\tilde{t})-\frac{\alpha \kappa e^{-R^{2}} \omega_{N} R^{N-1}}{2 N \pi^{N / 2}}\right) e^{2 N(1-p) \tilde{t}}
$$

Thus for $t>\tilde{t} \geq 0$,

$$
F^{p-1}(t) \geq\left[\frac{\alpha \kappa e^{-R^{2}} \omega_{N} R^{N-1}}{2 N \pi^{N / 2}}+\left(F^{1-p}(\tilde{t})-\frac{\alpha \kappa e^{-R^{2}} \omega_{N} R^{N-1}}{2 N \pi^{N / 2}}\right) e^{2 N(p-1)(t-\tilde{t})}\right]^{-1} .
$$

We would like to show that there exists $\tilde{t}$ such that

$$
F^{1-p}(\tilde{t})-\frac{\alpha \kappa e^{-R^{2}} \omega_{N} R^{N-1}}{2 N \pi^{N / 2}}<0 .
$$


From (2.2) and (2.6),

$$
\begin{aligned}
u(x, t) & \geq \alpha \kappa \int_{0}^{t} \int_{\partial B} g(x, t ; \xi, \tau) u^{p}(\xi, \tau) d S_{\xi} d \tau \\
& =\alpha \kappa \int_{0}^{t} \int_{\partial B} g(x, t ; \xi, \tau) M^{p}(\tau) d S_{\xi} d \tau .
\end{aligned}
$$

For $t>1$,

$$
u(x, t) \geq \alpha \kappa \int_{0}^{t-1} M^{p}(\tau) \int_{\partial B} g(x, t ; \xi, \tau) d S_{\xi} d \tau .
$$

Since $u$ is a nondecreasing function of $t$, we have $M^{p}(\tau) \geq M^{p}(0)>0$. Thus,

$$
\begin{aligned}
u(x, t) & \geq \alpha \kappa M^{p}(0) \int_{0}^{t-1} \int_{\partial B} g(x, t ; \xi, \tau) d S_{\xi} d \tau \\
& =\alpha \kappa M^{p}(0) \int_{0}^{t-1} I(x, t-\tau) d \tau \\
& =\alpha \kappa M^{p}(0) \int_{1}^{t} I(x, \theta) d \theta .
\end{aligned}
$$

Using (3.1), we have for any $x \in \bar{B}$,

$$
\begin{aligned}
u(x, t) & \geq \alpha \kappa M^{p}(0)(4 \pi)^{-N / 2} e^{-R^{2}} \omega_{N} R^{N-1} \int_{1}^{t} \theta^{-N / 2} d \theta \\
& =\left\{\begin{array}{l}
2 \alpha \kappa M^{p}(0)(4 \pi)^{-N / 2} e^{-R^{2}} \omega_{N} R^{N-1}\left(t^{1 / 2}-1\right) \text { if } N=1, \\
\alpha \kappa M^{p}(0)(4 \pi)^{-N / 2} e^{-R^{2}} \omega_{N} R^{N-1} \ln t \text { if } N=2 .
\end{array}\right.
\end{aligned}
$$

Thus, there exists $\tilde{t}$ such that for $t \geq \tilde{t}$,

$$
u(x, t)>\frac{\left(2 N \pi^{N / 2}\right)^{1 /(p-1)}}{\alpha^{1 /(p-1)}\left(\kappa e^{-R^{2}} \omega_{N} R^{N-1}\right)^{1 /(p-1)}\left(\int_{\bar{B}} h(x) d x\right)}
$$

for any $x \in \bar{B}$. Then,

$$
\begin{aligned}
F^{p-1}(\tilde{t}) & =\left(\int_{\mathbb{R}^{N}} u(x, \tilde{t}) h(x) d x\right)^{p-1} \\
& \geq\left(\int_{\bar{B}} u(x, \tilde{t}) h(x) d x\right)^{p-1} \\
& >\left[\frac{\left(2 N \pi^{N / 2}\right)^{1 /(p-1)}}{\alpha^{1 /(p-1)}\left(\kappa e^{-R^{2}} \omega_{N} R^{N-1}\right)^{1 /(p-1)}\left(\int_{\bar{B}} h(x) d x\right)}\right]^{p-1}\left(\int_{\bar{B}} h(x) d x\right)^{p-1} \\
& =\frac{2 N \pi^{N / 2}}{\alpha \kappa e^{-R^{2}} \omega_{N} R^{N-1}},
\end{aligned}
$$

which gives (3.4). From (3.3), there exists a finite time $t_{b}(>\tilde{t})$ such that $\lim _{t \rightarrow t_{b}} F(t)=$ $\infty$. Thus, $u(x, t)$ blows up in a finite time. 
4. $N \geq 3$. In this section, we show that the blow-up behavior for $N \geq 3$ is completely different from that for $N \leq 2$.

Theorem 4.1. (i) For $N \geq 3$, if $\alpha$ is sufficiently small, then the solution $u$ of the problem (1.1) exists globally.

(ii) For $N \geq 3$, if $\alpha$ is sufficiently large, then the solution $u$ of the problem (1.1) blows up in a finite time.

Proof. (i) Since $f^{\prime}(u)>0$, we have $f(u) \leq f(2 M(0))$ for $u(x, t) \leq 2 M(0)$. Thus for $u(x, t) \leq 2 M(0)$, it follows from (2.2) and $\int_{\mathbb{R}^{N}} g(x, t ; \xi, 0) d \xi=1$ (cf. Evans [5, p. 46]) that

$$
\begin{aligned}
u(x, t) & \leq M(0) \int_{\mathbb{R}^{N}} g(x, t ; \xi, 0) d \xi+\alpha \int_{0}^{t} \int_{\partial B} g(x, t ; \xi, \tau) f(2 M(0)) d S_{\xi} d \tau \\
& =M(0)+\alpha f(2 M(0)) \int_{0}^{t} \int_{\partial B} g(x, t ; \xi, \tau) d S_{\xi} d \tau .
\end{aligned}
$$

Let $\eta=\left(\xi_{i}-x_{i}\right) /(2 \sqrt{t-\tau})$. Using $\int_{-\infty}^{\infty} e^{-\eta^{2}} d \eta=\sqrt{\pi}$, we have

$$
\int_{\partial B} g(x, t ; \xi, \tau) d S_{\xi} \leq \frac{1}{2 \sqrt{\pi}(t-\tau)^{1 / 2}} .
$$

For $0<t \leq 1$, we have

$$
\begin{aligned}
u(x, t) & \leq M(0)+\frac{\alpha f(2 M(0))}{2 \sqrt{\pi}} \int_{0}^{t} \frac{d \tau}{(t-\tau)^{1 / 2}} \\
& \leq M(0)+\frac{\alpha f(2 M(0))}{\sqrt{\pi}} .
\end{aligned}
$$

For $t>1$, and for any $b \in \partial B$,

$$
\begin{aligned}
u(x, t) & \leq u(b, t) \\
& \leq M(0)+\alpha f(2 M(0))\left(\int_{0}^{t-1} \int_{\partial B} g(b, t ; \xi, \tau) d S_{\xi} d \tau+\int_{t-1}^{t} \int_{\partial B} g(b, t ; \xi, \tau) d S_{\xi} d \tau\right) \\
& \leq M(0)+\alpha f(2 M(0))\left(\int_{0}^{t-1} I(b, t-\tau) d \tau+\int_{t-1}^{t} \frac{d \tau}{2 \sqrt{\pi}(t-\tau)^{1 / 2}}\right) \\
& =M(0)+\alpha f(2 M(0))\left(\int_{1}^{t} I(b, \theta) d \theta+\frac{1}{\sqrt{\pi}}\right) \\
& \leq M(0)+\alpha f(2 M(0))\left(\int_{1}^{\infty} I(b, \theta) d \theta+\frac{1}{\sqrt{\pi}}\right) .
\end{aligned}
$$

Using (3.1), we have for $N \geq 3$,

$$
\begin{aligned}
\int_{1}^{\infty} I(b, \theta) d \theta & \leq(4 \pi)^{-N / 2} \omega_{N} R^{N-1} \int_{1}^{\infty} \theta^{-N / 2} d \theta \\
& =\frac{(4 \pi)^{-N / 2} \omega_{N} R^{N-1}}{N / 2-1}<\infty .
\end{aligned}
$$

Thus, we can choose $\alpha(>0)$ sufficiently small such that the right-hand side of (4.1) is less than or equal to $2 M(0)$. Hence, the solution $u$ of the problem (1.1) exists globally. 
(ii) Let $\tilde{t}=0$ in (3.3). We have

$$
F^{p-1}(t) \geq\left[\frac{\alpha \kappa e^{-R^{2}} \omega_{N} R^{N-1}}{2 N \pi^{N / 2}}+\left(F^{1-p}(0)-\frac{\alpha \kappa e^{-R^{2}} \omega_{N} R^{N-1}}{2 N \pi^{N / 2}}\right) e^{2 N(p-1) t}\right]^{-1} .
$$

We note that $F(0)=\int_{\mathbb{R}^{N}} \psi(x) h(x) d x$. We would like to choose $\alpha$ sufficiently large such that $F^{1-p}(0)-\alpha \kappa e^{-R^{2}} \omega_{N} R^{N-1} /\left(2 N \pi^{N / 2}\right)<0$. This can be accomplished by choosing

$$
\alpha>\frac{2 N \pi^{N / 2} F^{1-p}(0)}{\kappa e^{-R^{2}} \omega_{N} R^{N-1}} .
$$

Thus, there exists a finite time $t_{b}$ such that $\lim _{t \rightarrow t_{b}} F(t)=\infty$ and hence $u(x, t)$ blows up in a finite time.

Let $k$ denote the positive constant $\int_{\mathbb{R}^{N}} \psi(\xi) d \xi$. Then,

$$
\int_{\mathbb{R}^{N}} \exp \left(-\frac{|x-\xi|^{2}}{4 t}\right) \psi(\xi) d \xi \leq \int_{\mathbb{R}^{N}} \psi(\xi) d \xi=k .
$$

We have

$$
\begin{aligned}
\int_{\mathbb{R}^{N}} g(x, t ; \xi, 0) \psi(\xi) d \xi & =\frac{1}{(4 \pi t)^{N / 2}} \int_{\mathbb{R}^{N}} \exp \left(-\frac{|x-\xi|^{2}}{4 t}\right) \psi(\xi) d \xi \\
& \leq \frac{k}{(4 \pi t)^{N / 2}},
\end{aligned}
$$

which tends to 0 as $t \rightarrow \infty$. This shows that the initial data do not affect the solution as $t$ tends to infinity. The fundamental solution (cf. Evans [5, pp. 22 and 615]) of the Laplace equation for $N \geq 3$ is given by

$$
G(x)=\frac{\Gamma\left(\frac{N}{2}+1\right)}{N(N-2) \pi^{N / 2}} \frac{1}{|x|^{N-2}} .
$$

The proof of the following result is the same as that of Theorem 4.2 of Chan and Tragoonsirisak [4.

Theorem 4.2. If $u(x, t) \leq C$ for some positive constant $C$, then $u(x, t)$ converges from below to a solution $U(x)=\lim _{t \rightarrow \infty} u(x, t)$ of the nonlinear integral equation,

$$
U(x)=\alpha \int_{\partial B} G(x-\xi) f(U(\xi)) d S_{\xi} .
$$

The next result shows that there exists a critical value for $\alpha$.

Theorem 4.3. For $N \geq 3$, there exists a unique $\alpha^{*}$ such that $u$ exists globally for $\alpha<\alpha^{*}$, and $u$ blows up in a finite time for $\alpha>\alpha^{*}$.

Proof. To show that the larger the $\alpha$, the larger the solution, let $\alpha>\beta$, and consider the sequence $\left\{v_{n}\right\}$ given by $v_{0}(x, t)=\psi(x)$, and for $n=0,1,2, \ldots$,

$$
v_{n+1}(x, t)=\int_{\mathbb{R}^{N}} g(x, t ; \xi, 0) \psi(\xi) d \xi+\beta \int_{0}^{t} \int_{\partial B} g(x, t ; \xi, \tau) f\left(v_{n}(\xi, \tau)\right) d S_{\xi} d \tau .
$$


Similar to the construction of the sequence $\left\{u_{n}\right\}$ in $\Omega$ in the proof of Theorem 2.4, we obtain

$$
\begin{aligned}
v(x, t) & =\lim _{n \rightarrow \infty} v_{n}(x, t) \\
& =\int_{\mathbb{R}^{N}} g(x, t ; \xi, 0) \psi(\xi) d \xi+\beta \int_{0}^{t} \int_{\partial B} g(x, t ; \xi, \tau) f(v(\xi, \tau)) d S_{\xi} d \tau .
\end{aligned}
$$

Since $u_{n}>v_{n}$ for $n=1,2,3, \ldots$, we have $u \geq v$. Hence, the solution $u$ is a nondecreasing function of $\alpha$. It follows from Theorem 4.1 that there exists a unique $\alpha^{*}$ such that $u$ exists globally for $\alpha<\alpha^{*}$ and $u$ blows up in a finite time for $\alpha>\alpha^{*}$.

We note that the critical value $\alpha^{*}$ is determined as the supremum of all positive values $\alpha$ for which a solution $U$ of (4.2) exists. The proof of the next result (showing that the solution $u$ exists globally when $\alpha=\alpha^{*}$ ) for the case $f(0)>0$ is a modification of that for Theorem 7 of Chan and Jiang [1] for a degenerate one-dimensional problem in a bounded domain.

TheOREM 4.4. For $N \geq 3$,

$$
\alpha^{*}=\frac{(N-2) \pi^{(N-3) / 2}}{R \Gamma\left(\frac{N-1}{2}\right) \prod_{i=1}^{N-3} \int_{0}^{\pi} \sin ^{i} \varphi d \varphi} \sup _{M(0)<s<\infty}\left(\frac{s}{f(s)}\right),
$$

where for $N=3, \prod_{i=1}^{N-3} \int_{0}^{\pi} \sin ^{i} \varphi d \varphi=1$. Furthermore, $u$ does not blow up in infinite time.

Proof. From (2.6), $U(x)=\lim _{t \rightarrow \infty} u(x, t)$ attains its maximum at $b \in \partial B$. From (4.2),

Thus,

and hence,

$$
U(b)=\alpha \int_{\partial B} G(b-\xi) f(U(b)) d S_{\xi} .
$$

$$
\alpha=\left(\frac{1}{\int_{\partial B} G(b-\xi) d S_{\xi}}\right)\left(\frac{U(b)}{f(U(b))}\right)
$$

$$
\alpha^{*}=\left(\frac{1}{\int_{\partial B} G(b-\xi) d S_{\xi}}\right) \sup _{M(0)<s<\infty}\left(\frac{s}{f(s)}\right) .
$$

From the proof of Theorem 4.5 of Chan and Tragoonsirisak [4],

$$
\int_{\partial B} G(b-\xi) d S_{\xi}=\frac{R \Gamma\left(\frac{N-1}{2}\right) \prod_{i=1}^{N-3} \int_{0}^{\pi} \sin ^{i} \varphi d \varphi}{(N-2) \pi^{(N-3) / 2}} .
$$

Thus, we have (4.3).

Let us consider the function $\varphi(s)=s / f(s)$.

CASE 1. If $f(0)=0$, then we claim that $\varphi(s)$ is a decreasing function for $s>0$. Since $f$ is a convex function (cf. Stromberg [11, p. 199]) in $(0, \infty)$, we have for any $0<s<s_{2}$,

$$
f\left((1-t) s+t s_{2}\right) \leq(1-t) f(s)+t f\left(s_{2}\right), t \in[0,1] .
$$

Letting $s \rightarrow 0$, we have

$$
f\left(t s_{2}\right) \leq t f\left(s_{2}\right) .
$$


Let $t=s_{1} / s_{2}$, where $0<s_{1}<s_{2}$. Then,

$$
f\left(s_{1}\right) \leq \frac{s_{1}}{s_{2}} f\left(s_{2}\right)
$$

which gives

$$
\varphi\left(s_{2}\right) \leq \varphi\left(s_{1}\right)
$$

implying that $\varphi(s)$ is a nonincreasing function of $s(>0)$. It follows from (4.3) that

$$
\alpha^{*}=\frac{(N-2) \pi^{(N-3) / 2}}{R \Gamma\left(\frac{N-1}{2}\right) \prod_{i=1}^{N-3} \int_{0}^{\pi} \sin ^{i} \varphi d \varphi}\left(\frac{M(0)}{f(M(0))}\right) .
$$

CASE 2. If $f(0)>0$, then $\varphi(s)>0$ for $s>0$, and $\varphi(0)=0=\lim _{s \rightarrow \infty} \varphi(s)$. We have $\varphi^{\prime}(s)=\left(f(s)-s f^{\prime}(s)\right) / f^{2}(s)$. Therefore, a relative maximum or minimum occurs at $\tilde{s} \in(0, \infty)$, where $f(\tilde{s})=\tilde{s} f^{\prime}(\tilde{s})$. Since $\varphi^{\prime \prime}(\tilde{s})=-\tilde{s} f^{\prime \prime}(\tilde{s}) / f^{2}(\tilde{s})<0, \varphi(s)$ attains its absolute maximum when $\varphi(\tilde{s})=1 / f^{\prime}(\tilde{s})$. Thus, $\sup _{0<s<\infty}(s / f(s))$ occurs at $s=\tilde{s} \in(0, \infty)$. We note that the function $\varphi(s)$ is a strictly increasing function for $0 \leq s<\tilde{s}$, and a strictly decreasing function for $s>\tilde{s}$. Thus, if $M(0)<\tilde{s}$, then

$$
\alpha^{*}=\frac{(N-2) \pi^{(N-3) / 2}}{R \Gamma\left(\frac{N-1}{2}\right) \prod_{i=1}^{N-3} \int_{0}^{\pi} \sin ^{i} \varphi d \varphi}\left(\frac{\tilde{s}}{f(\tilde{s})}\right) .
$$

If $M(0) \geq \tilde{s}$, then it follows from $\varphi(s)$ being a strictly decreasing function for $s>\tilde{s}$ that $\varphi(s)$ attains its supremum at $M(0)$. Thus,

$$
\alpha^{*}=\frac{(N-2) \pi^{(N-3) / 2}}{R \Gamma\left(\frac{N-1}{2}\right) \prod_{i=1}^{N-3} \int_{0}^{\pi} \sin ^{i} \varphi d \varphi}\left(\frac{M(0)}{f(M(0))}\right) .
$$

From (4.4) to (4.6), $\alpha^{*}$ occurs at some finite positive value. Hence for $\alpha \leq \alpha^{*}, u$ exists globally. Since $u$ blows up in a finite time for $\alpha>\alpha^{*}, u$ does not blow up in infinite time.

For an illustration, we give below two examples on calculating $\alpha^{*}$ for some given functions $f$ and some given initial data on the surface of the ball $M(0)$.

EXAmple 4.5. Let $f(u)=u^{p}$. Since $f(0)=0$, it follows from (4.4) that

$$
\alpha^{*}=\frac{(N-2) \pi^{(N-3) / 2}}{M^{p-1}(0) R \Gamma\left(\frac{N-1}{2}\right) \prod_{i=1}^{N-3} \int_{0}^{\pi} \sin ^{i} \varphi d \varphi} .
$$


Example 4.6. Let $f(u)=(u+1)^{p}$. Since $f(0)>0$, we have $\tilde{s}=1 /(p-1)$. From (4.5) and (4.6),

$$
\alpha^{*}= \begin{cases}\frac{(p-1)^{p-1}(N-2) \pi^{(N-3) / 2}}{p^{p} R \Gamma\left(\frac{N-1}{2}\right) \prod_{i=1}^{N-3} \int_{0}^{\pi} \sin ^{i} \varphi d \varphi} & \text { if } M(0)<\frac{1}{p-1}, \\ \frac{M(0)(N-2) \pi^{(N-3) / 2}}{(M(0)+1)^{p} R \Gamma\left(\frac{N-1}{2}\right) \prod_{i=1}^{N-3} \int_{0}^{\pi} \sin ^{i} \varphi d \varphi} & \text { if } M(0) \geq \frac{1}{p-1} .\end{cases}
$$

\section{REFERENCES}

[1] C. Y. Chan, X. O. Jiang, Quenching for a degenerate parabolic problem due to a concentrated nonlinear source, Quart. Appl. Math. 62 (2004), 553-568. MR.2086046 (2005e:35139)

[2] C. Y. Chan, H. Y. Tian, Multi-dimensional explosion due to a concentrated nonlinear source, J. Math. Anal. Appl. 295 (2004), 174-190. MR2064418 (2005d:35115)

[3] C. Y. Chan, H. Y. Tian, A criterion for a multi-dimensional explosion due to a concentrated nonlinear source, Appl. Math. Lett. 19 (2006), 298-302. MR2202419 (2006h:35118)

[4] C. Y. Chan, P. Tragoonsirisak, A multi-dimensional quenching problem due to a concentrated nonlinear source in $\mathbb{R}^{N}$, Nonlinear Anal. 69 (2008), 1494-1514. MR2424525 (2009g:35128)

[5] L. C. Evans, Partial Differential Equations, Graduate Studies in Mathematics, vol. 19, American Mathematical Society, Providence, RI, 1998, pp. 22, 46, 615. MR.1625845 (99e:35001)

[6] A. Friedman, Partial Differential Equations of Parabolic Type, Prentice Hall, Englewood Cliffs, NJ, 1964, pp. 34, 49, 74. MR0181836 (31:6062)

[7] H. Fujita, On the blowing up of solutions of the Cauchy problem for $u_{t}=\Delta u+u^{1+\alpha}$, J. Fac. Sci. Univ. Tokyo Sect. I 13 (1966), 109-124. MR0214914 (35:5761)

[8] R. G. Pinsky, Existence and nonexistence of global solutions for $u_{t}=\Delta u+a(x) u^{p}$ in $R^{d}$, J. Differential Equations 133 (1997), 152-177. MR1426761 (97k:35118)

[9] M. H. Protter, H. F. Weinberger, Maximum Principles in Differential Equations, Prentice-Hall, Englewood Cliffs, NJ, 1967, pp. 183-185. MR0219861 (36:2935)

[10] I. Stakgold, Boundary Value Problems of Mathematical Physics, vol. II, Macmillan, New York, NY, 1968, p. 198. MR0243183(39:4507)

[11] K. R. Stromberg, An Introduction to Classical Real Analysis, Wadsworth, Belmont, CA, 1981, p. 199. MR0604364 (82c:26002) 